\title{
Temperature and Field Dependence of Field Grading Tubes for Medium Voltage XLPE Cable Joints
}

\author{
Frank Mauseth \\ Norwegian Univ. of Science and Technology \\ Dept. of Electric Power Engineering \\ Trondheim, Norway
}

\begin{abstract}
In Norway it has been observed that many medium voltage (12 and $24 \mathrm{kV}$ ) cable sections with heat shrink joints have suffered from overheating often causing failure during service. The overheating is due to a bad metallic connection between the aluminium conductors in the joint. This is especially observed for XLPE cables installed in the 80's, which constitutes a significant part of the installed cables in the network.

This paper is part of a work to elucidate the mechanisms causing a low insulation resistance of MV cable joints. As a bad metallic connection can result in local high temperatures during normal operation, the properties of a heat shrink stress control tubes commonly used in such joints has been characterized at temperatures around the melting peak of the material that is just above the maximum service temperature of $90{ }^{\circ} \mathrm{C}$ for XLPE cables. The main purpose of this paper is to examine the effect of these temperatures on the electrical properties of the field grading material. Here, the electrical properties have been characterized by time domain dielectric response measurements at different temperatures and axial electrical fields.

The results show that the conductivity of the stress control tube is temperature dependent. Also, a polarity change in the depolarization current at higher electrical field strengths was observed.
\end{abstract}

\section{Introduction}

Failure statistics for medium voltage cable in Norway show that the overall failure rates for the cable accessories are relatively low [1]. However, for some types of joints and especially those installed in the 1980 's, the electrical conductivity is becoming very high and such joints have an increasing failure rate. From dissections of joints suffered from breakdown, it has been found that some of the failures have been caused by thermal degradation likely due to a too high transition resistance of the metal connector [1]. This has resulted in a severe ageing of the joint insulation, leading to embrittlement and cracking of the insulation material initiating partial discharges.

The main purpose of this work has been to investigate the dielectric properties of the field grading tube of cable joints. As bad metallic connections is likely to lead to local high temperatures exceeding $90{ }^{\circ} \mathrm{C}$, the maximum service temperature of XLPE cables, the

\author{
Henrik Enoksen, Sverre Hvidsten \\ SINTEF Energy Research \\ Trondheim, Norway
}

properties of a heat shrink field grading tube has been investigated.

\section{Background}

When applying a DC voltage across a dielectric material, a polarization current will flow through the insulation as a result of the conductivity and the dielectric displacement. This current can be expressed by:

$$
I_{p}(t)=C_{0} U\left(\frac{\sigma}{\epsilon_{0}}+f(t)\right)
$$

where $C_{0}$ is the geometric capacitance of the dielectric material, $U$ is the applied voltage, $\sigma$ is the conductivity, $\epsilon_{0}$ is the vacuum permittivity and $f(t)$ is the dielectric response function of the material [2]. After some time, the dielectric material is fully polarized and the contribution from the dielectric response function vanishes. Thus, at this state, only the conductivity will give a contribution to the current flowing through the dielectric.

Short-circuiting the dielectric material gives rise to a depolarization current that flows in the opposite direction of the polarization current. The depolarization current can be expressed as:

$$
I_{d}(t)=C_{0} U\left(f(t)-f\left(t+t_{c}\right)\right)
$$

where $f\left(t+t_{c}\right)$ denotes remaining charges in the dielectric from previous voltage applications. As no voltage is applied across the dielectric during this period, there will be no contribution form the conductivity.

Assuming $f\left(t+t_{c}\right)$ is small, (1) and (2) can be combined and the conductivity of the material can be expressed as:

$$
\sigma \approx \frac{\epsilon_{0}}{C_{0} U}\left(I_{p}(t)-I_{d}(t)\right)
$$

where $\epsilon_{0}$ is the vacuum permittivity [2].

The working principle of a field grading material is the field dependent conductivity (non-linearity). At low electrical fields, the conductivity is relatively low, but typically increases strongly from a low to a high conductivity in a narrow electrical field region. When using field grading materials at e.g. a termination, the 
non-linear properties are used to reduce the high fields around the edge of the outer semi-conductor, as space charges forms in the field grading material creating a counter field [3].

\section{Experimental Work}

\subsection{Test objects}

In this study, a commercially available heat shrink stress control tube was used. The tube is normally used in medium voltage cable joints with shrinking insulation tube of rubber (EPDM or EPR) and an outer semiconductor. In addition, mastic is also used filling the gap between the conductor joint and the field grading material. Here, only the field grading tube has been used.

In the experiments, the stress control tube was installed on a polytetrafluoroethylene (PTFE) rod with metallic electrodes by heat shrinking. The electrodes were attached at each side as indicated in Fig. 1. The outer diameter of the test object after installation was $29 \mathrm{~mm}$, that is within the recommended range when installing heat shrink joints in service.

The sample lengths of the PTFE rod used in the experiments was $20 \mathrm{~mm}$. To ensure a good electric contact, metallic clamps were used around each end of the stress control tube. To avoid any corona discharges, toroids were attached at both ends. At the ground side, the toroid was grounded and insulated from the measuring electrode on the ground side of the stress control tube. The final assembly of the test object is illustrated in Fig. 1.

The test object was pre-conditioned in a vacuum chamber at $70{ }^{\circ} \mathrm{C}$ for three days before performing the measurements.

\subsection{Differential Scanning Calorimetry}

Differential scanning calorimetry (DSC) measurements were performed from 20 to $200^{\circ} \mathrm{C}$ at a rate of $10^{\circ} \mathrm{C} / \mathrm{min}$. The measurements were performed two times to study the phase transitions the stress control sleeve material undergoes in the temperature interval used in the measurements. These measurements were performed on small samples with a diameter of $3 \mathrm{~mm}$ that were 1) fully shrunk, 2) virgin (i.e. not shrunk), and 3) partially shrunk to only fit the outer diameter of the

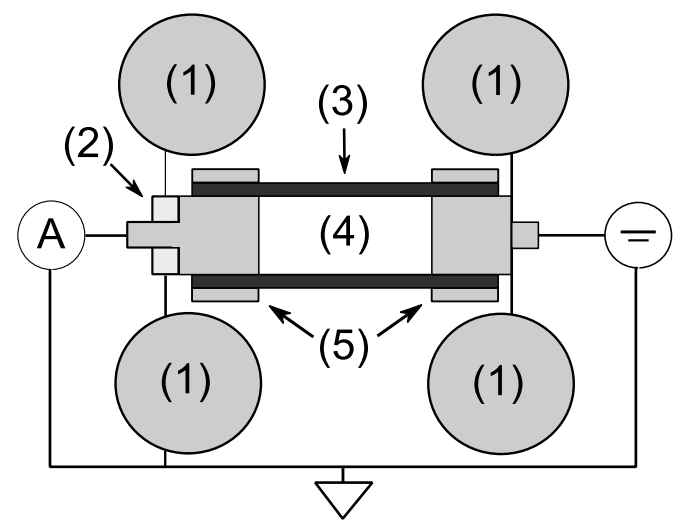

PTFE rod.

\subsection{Breakdown Voltage}

In order to examine whether conductive heating may occur at the electrical stress levels used during the measurements, a DC breakdown voltage step-up test was performed. The DC voltage was increased in steps of $5 \mathrm{kV}$ every 15 minute, from $5 \mathrm{kV}$ to $30 \mathrm{kV}$ or until breakdown occurred. Using an infrared camera, the temperature was measured every five minutes at the end of each voltage step. The temperature sensitivity of the camera was ca. $0.1^{\circ} \mathrm{C}$.

\subsection{Time Domain Dielectric Response}

Polarization and depolarization currents were measured for one hour each at the voltages listed in Table 1. This table also shows the corresponding mean electric fields across the test object. After the measurement at $20 \mathrm{kV}$, a second measurement at $1 \mathrm{kV}$ was performed to examine the reproducibility of the measurements. The measurements were performed at $90{ }^{\circ} \mathrm{C}$, and the melting onset, peak and offset temperatures found from the DSC measurements. Prior to starting the measurements, the sample was temperature conditioned for one hour. The experimental set-up, consisting of a highly stable high voltage DC source and a picoamperemeter, is shown in Fig. 1.

In order to examine the influence of the PTFE rod on the measurements, polarization and depolarization currents were also measured without the stress control sleeve at 1,10 and $15 \mathrm{kV}$ at 40 and $150{ }^{\circ} \mathrm{C}$.

Table 1 - Average electric fields in $\mathrm{kV} / \mathrm{mm}$ for the test specimens for the applied voltages.

\begin{tabular}{|l|l|l|l|l|l|l|l|l|}
\hline$U[\mathrm{kV}]$ & 0.5 & 1 & 2.5 & 5 & 10 & 12.5 & 15 & 20 \\
\hline $\begin{array}{l}E \\
{[\mathrm{kV} / \mathrm{mm}]}\end{array}$ & 0.025 & 0.05 & 0.125 & 0.25 & 0.5 & 0.62 & 0.75 & 1.0 \\
\hline
\end{tabular}

\section{Results and Discussion}

\subsection{Differential scanning calorimetry}

Fig. 2 shows the first and second heating run for the stress control tube sample taken from the test object

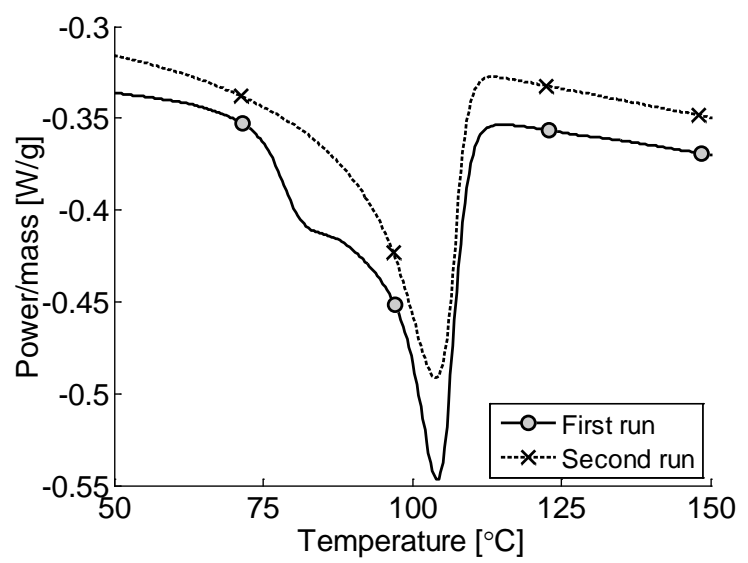

Fig. 2 - DSC measurements for a partially shrunk stress control tube.

Fig. 1 - Test object used in the experiments; (1) toroids, (2) PTFE piece insulating the measuring electrode from the grounded corona ring, (3) stress control tube, (4) $\$$ PTenmark, Copenhagen, Denmark 15. -17. June, 2015 and (5) metallic clamps. 
(partially shrunk). It can be seen that the stress control sleeve has a melting peak $\left(T_{m}\right)$ at approximately $103^{\circ} \mathrm{C}$. The onset of this peak $\left(T_{o n}\right)$ is abound $94^{\circ} \mathrm{C}$, which is just above the maximum allowed service temperature $\left(90^{\circ} \mathrm{C}\right)$ of $\mathrm{XLPE}$ cables. The offset temperature $\left(T_{\text {off }}\right)$ was found to be $113^{\circ} \mathrm{C}$. The local peak at about $80^{\circ} \mathrm{C}$ is also present for the virgin sleeve, and thus the origin of this peak is an incomplete shrinking. No such peak is observed for the fully shrunk sample. For this sample, the first and second heating runs are identical.

\subsection{DC breakdown voltage of the test samples}

No conductive heating was observed for the sample at electric fields up to $1 \mathrm{kV} / \mathrm{mm}$. When increasing the electric field to $1.25 \mathrm{kV} / \mathrm{mm}$, a surface breakdown occurred. This is most likely caused by the lack of corona rings, leading to an uneven field distribution along the tube. The infrared measurements show that the stress control sleeve did not experience any additional heating in the electric field range used here. Any self-heating effects during the polarization current measurements at low temperatures $\left(40^{\circ} \mathrm{C}\right.$ and below) can therefore to be neglected.

\subsection{Time Domain Dielectric Response}

The currents measured on the PTFE sample without any stress control tube resulted in noise signals only (currents below $1 \mathrm{pA}$ ). Consequently, the PTFE rod does not contribute to the measured dielectric response.

Fig. 3 shows the polarization currents for the test objects with stress control tubes at the lowest applied electrical field $0.05 \mathrm{kV} / \mathrm{mm}$, and the highest field value $1 \mathrm{kV} / \mathrm{mm}$, and temperatures from 90 to $113^{\circ} \mathrm{C}$. As can be seen, the currents depend on the temperature. Also the field dependency can be seen in the graphs, as the current increases by a magnitude of more than one when increasing the average field stress from 0.05 to $1 \mathrm{kV} / \mathrm{mm}$.

The currents do not change much with time, indicating that the current contribution from the polarization mechanisms is low compared to the conductive contribution to the currents.

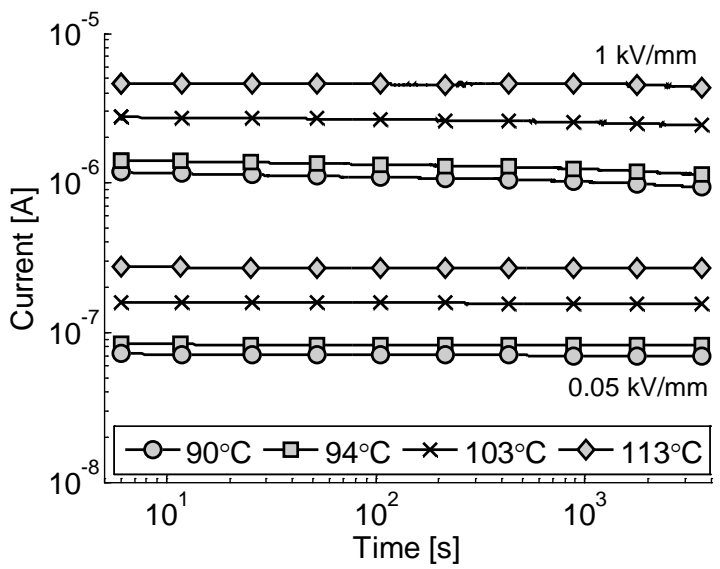

Fig. 3 - Polarization current as function of time at different temperatures and 0.05 and $1 \mathrm{kV} / \mathrm{mm}$.

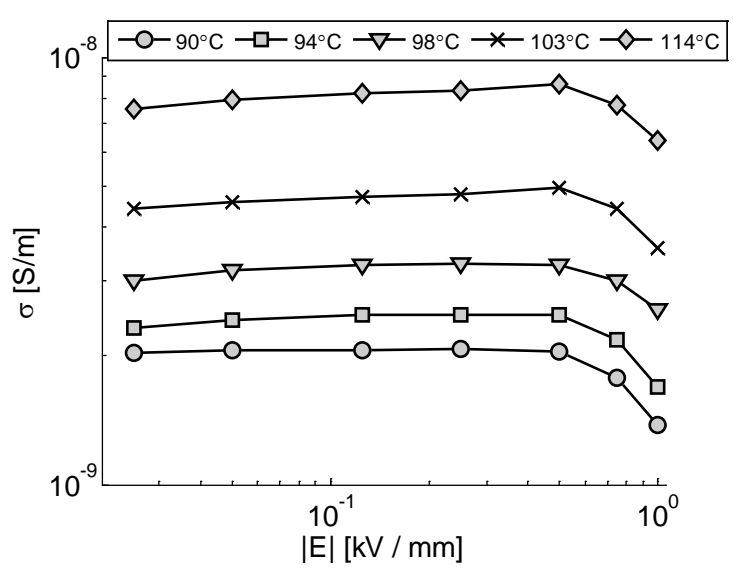

Fig. 4 - Conductivity as function of electric field for different temperatures.

Using (3), the conductivity was calculated and is shown in Fig. 4 as function of electric field for the different temperatures. The mean value of the last two minutes of the polarization and depolarization currents is used to calculate the conductivity. Up to field strengths of $0.4 \mathrm{kV} / \mathrm{mm}$, the conductivity is approximately independent of the electric field, while when applying a

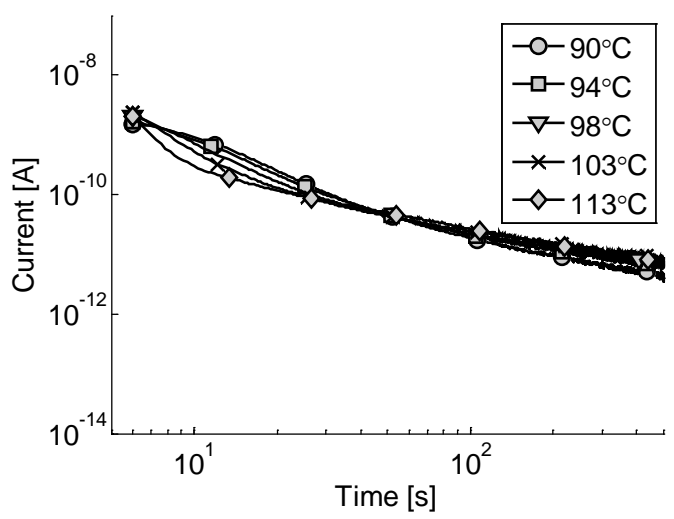

Fig. 5 - Depolarization currents at $1 \mathrm{kV}(0.05 \mathrm{kV} / \mathrm{mm})$ at different temperatures.

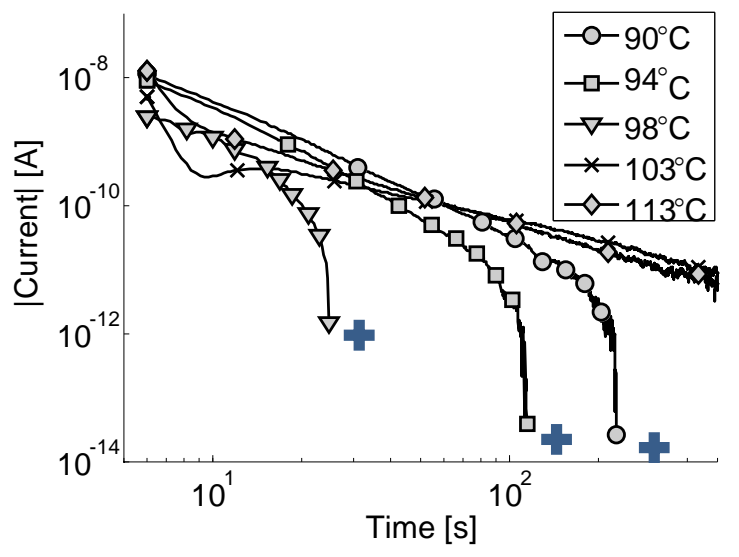

Fig. 6 - Depolarization currents at $20 \mathrm{kV}(1 \mathrm{kV} / \mathrm{mm})$ at different temperatures. For the three lowest temperatures, the current changes polarity from negative to positive (marked with a "+" in the graphs). The currents at 103 and $113^{\circ} \mathrm{C}$ are positive) 


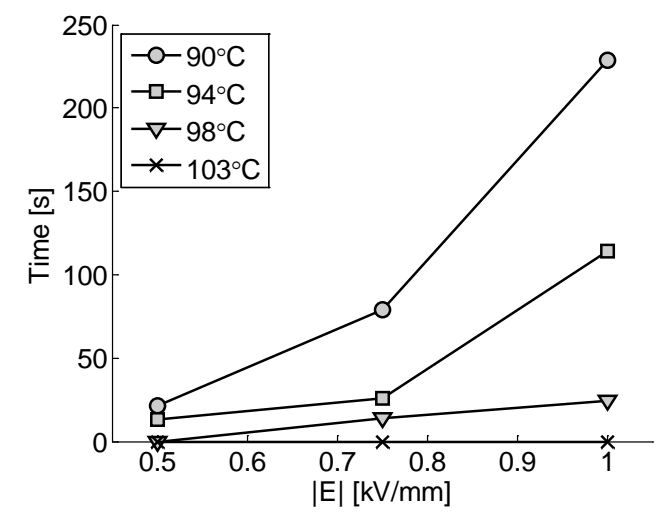

Fig. 7 - Time to polarity shift from negative to positive for the depolarization currents at different temperatures. At temperatures at melting peak $\left(103^{\circ} \mathrm{C}\right)$ and higher, the depolarization currents starts at a positive value.

higher field, a small decrease in the conductivity is observed. The small decrease in the conductivity might be a result of the relatively short polarization time of 1 hour and the effect being more pronounced at a higher field level.

In Fig. 5 and 6, the depolarization currents at 0.05 and $1 \mathrm{kV} / \mathrm{mm}$ are shown respectively. At the lowest field strength, the depolarization currents start at a positive value and follow an exponential decrease for all temperatures in the experiments.

For the highest field level, the measurements for the lowest temperatures under the melting peak, show a decreasing negative depolarization current in the beginning. After some time, the depolarization current changes polarity from negative to positive and after reaching a top, decreases slowly towards zero for the rest of the measurement period. The change of polarity is also show in Fig. 7, where the time to the observed polarity change is shown as function of the average applied field strength for the temperature up to the melting peak. For temperatures from melting peak and higher, the depolarization current starts at a positive value for fields above $0.5 \mathrm{kV} / \mathrm{mm}$, and decreases towards zero. The decrease follows Curie von Schweidler's law after reaching a peak value. This type of current-time dependencies has earlier been observed in both low- and high-density polyethylene films at high temperature and high electric fields of typically 40 $\mathrm{kV} / \mathrm{mm}[5,6]$. The polarity change is believed to be caused by build-up of space charges consisting of electrons and holes.

The main difference from previously reported depolarization current measurements is that the average applied fields in this experiments are much lower. However, the local fields in the stress control sleeve can be much larger than the mean electric field due to the inorganic conducting fillers, and probably comparable to the fields reported in $[5,6]$.

A field grading material should have a conductivity at least 100 times that of the cable and joint body insulation to avoid any local field enhancements [3]. However, there is a threshold of how high the conductivity can be. A higher conductivity leads to larger currents through the material from conductor to ground, causing increased ohmic losses. As the conductivity increases with the temperature, the ohmic heating can lead to increased ageing that again is likely to lead to increased conductivity, with an increased risk of thermal breakdown as result.

However, other factors like absorbed water will also contribute to increase the conductivity [7]. If the field grading tube does not connect the ground potential of the outer insulation screen and the high voltage metallic connector, the increased conductivity of the tube can cause critical field enhancement at edge of the tube.

\section{Conclusions}

From this work, the following conclusions can be made:

- The conductivity of the stress control tube is temperature dependent.

- The field dependency is low. However, a decrease in the conductivity was observed for the highest field strengths. This can be caused by the relative short polarization time of 1 hour.

- Polarity change in the depolarization current at field strengths above $0.5 \mathrm{kV} / \mathrm{mm}$ may indicate local space charge build up in the stress control tube even at low average electrical field strengths. This causes anomalous depolarization currents where the polarity is the same as for the polarization currents at temperatures at the melting peak and higher.

\section{References}

[1] H. Faremo et.al, " Service experience for XLPE cables installed in Norway - from graphite painted insulation screens to axially and radially water tight cable constructions," 14th Int. Conference and Exhibition on Electricity Distribution (CIRED), Birmingham, pp. 321-325, 1997.

[2] S. Hvidsten and J.T. Benjaminsen, "Diagnostic testing of MV cables with low density of water trees," IEEE Int. Sym. Elec. In., Anaheim CA, USA, April 2-5, 2000, pp. 105-108.

[3] A.K. Johnscher, "Dielectric relaxation in solids”, Chelsea Dielectric Press, London 1983.

[4] Werelius et.al, "Dielectric spectroscopy for diagnosis of water tree deterioration in xlpe cables,” IEEE Trans. Dielectr. Electr. Insul,vol. 8, no.1, February 2001, pp. 27-42.

[5] I. Kitani, Y. Tsuji, and K. Arii, 1984, "Analysis of Anomalous Discharge Current in LowDensity Polyethylene", Jpn. J. Appl. Phys., vol. 23, no. 7, 855-860.

[6] G. Chen, 2009, "Anomalous Phenomena in Solid Dielectrics under High Electric Fields", $9^{\text {th }}$ International Conference on Properties and Applications of Dielectric Materials, 954-960. 
[7] F. Mauseth et.al, "Effect of Water on Service Performance of Medium Voltage XLPE Cable Joints”, NordIS 2011, Tampere, Finland. 